\title{
Attitude Control of a Single Tilt Tri-Rotor UAV System: Dynamic Modeling and Each Channel's Nonlinear Controllers Design
}

\author{
Juing-Shian Chiou, ${ }^{1}$ Huu-Khoa Tran, ${ }^{2}$ and Shou-Tao Peng ${ }^{2}$ \\ ${ }^{1}$ Department of Electrical Engineering, Southern Taiwan University of Science and Technology, Tainan, Taiwan \\ ${ }^{2}$ Mechanical Engineering Department, Southern Taiwan University of Science and Technology, Tainan, Taiwan \\ Correspondence should be addressed to Juing-Shian Chiou; jschiou@mail.stust.edu.tw
}

Received 2 July 2013; Revised 20 August 2013; Accepted 27 August 2013

Academic Editor: Dan Wang

Copyright ( $\odot 2013$ Juing-Shian Chiou et al. This is an open access article distributed under the Creative Commons Attribution License, which permits unrestricted use, distribution, and reproduction in any medium, provided the original work is properly cited.

\begin{abstract}
This paper has implemented nonlinear control strategy for the single tilt tri-rotor aerial robot. Based on Newton-Euler's laws, the linear and nonlinear mathematical models of tri-rotor UAVs are obtained. A numerical analysis using Newton-Raphson method is chosen for finding hovering equilibrium point. Back-stepping nonlinear controller design is based on constructing Lyapunov candidate function for closed-loop system. By imitating the linguistic logic of human thought, fuzzy logic controllers (FLCs) are designed based on control rules and membership functions, which are much less rigid than the calculations computers generally perform. Effectiveness of the controllers design scheme is shown through nonlinear simulation model on each channel.
\end{abstract}

\section{Introduction}

The UAVs (Unmanned Aerial Vehicles)-which are defined by the U.S. Ministry of Defense as flying vehicles without pilots-have seen exponential growth in military surveillance, civilian information search, and rescue operations during the last decade. The development of UAVs requires a variety branch of knowledge including aeronautics, automatic control, computer science, signal processing, and sensors. One of current trends is to design the small flying machines capable of hovering maneuvers as well as forward flight. Though some dedicated efforts to improve the traditional helicopter structure frame, there are some new aerodynamical configurations that introduce important features [18]. VTOL (vertical takeoff and landing) UAV generally has shapes like helicopter or quadrotor. However, quadrotor UAV has more blades and more motors than general helicopter. So it is more dangerous, and its energy efficiency is low.

This paper presents a novel tri-rotor UAV system equipped with a single tilt servomotor on the tail part of the tri-rotor UAV to delete the inverse torque and enhance controllability of yaw moment. The other two fronts of rotors rotate in opposite directions, which created reaction torque almost zero. The proposed frame design is equidistant from gravity centre to stabilize itself automatically at any desired hovering tasks, which are calculated by NewtonRaphson method [9]. We have also proposed the backstepping control strategy which stabilizes via Lyapunov global stability theorem. Hence, the whole system is stable and controllable. Fuzzy logic, which makes it easier to mechanize tasks, has the advantage of a solution to the problem that human operators can understand; thus their experience can be used to design the controller.

\section{Dynamic Modeling of the Tri-Rotor UAV}

2.1. Single Tilt Tri-Rotor Model. The Newton-Euler formulation is used to develop the model of the tri-rotor. In Figure 1, which is derived from [6], the vehicle is described using a right hand generalized earth coordinate system of axes and a right hand body frame. Positive $x$-axis points towards the front rotors (rotors 1 and 2), positive $y$-axis points towards 

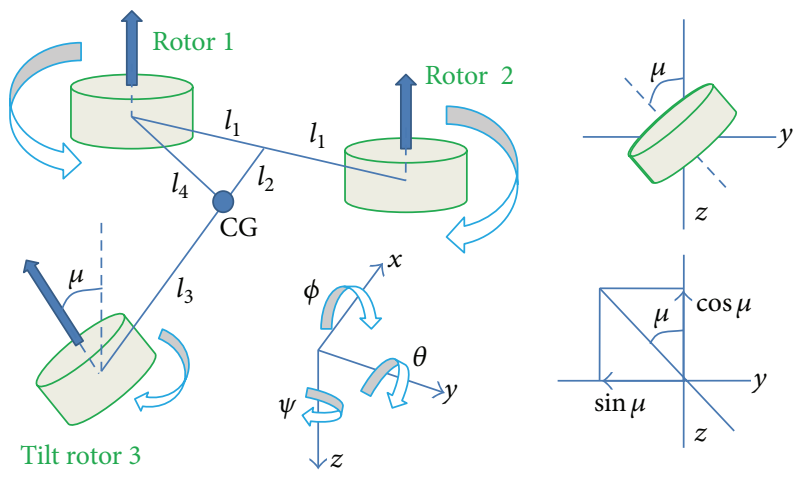

FIgURE 1: The tri-rotor configuration.

right (rotor 2), and positive $z$-axis is directed downwards. Positive sense of the three angular variables Roll $(\phi)$, Pitch $(\theta)$, and Yaw $(\psi)$ is decided by a right handed rotation about positive $x, y$, and $z$ axes, respectively. The tilt angle $\mu$ is measured by $y-z$ coordinate axis.

The dynamic modeling suggested of triple tilting rotor UAV is introduced based on Newton-Euler mathematical formulation, which has six degrees of freedom (DOF) and four inputs: three speeds of rotorcraft and one tilt angle. Following the conventional helicopter control commands, tri-rotor UAVs have similar commands, which are collective, lateral, longitudinal, and yaw or pedal $[4,10]$. They are indicated as $\delta$ col, $\delta$ lat (Roll control), $\delta$ lon (Pitch control), and $\delta$ ped (Yaw control). Since two front rotors are operated in different speeds, they generate the Roll $(\phi)$ control, for example, when speed of rotor 1 is up and that of rotor 2 down, and make the UAV toward the right and vice versa. The Pitch $(\theta)$ control is created as the third tail rotor changes velocity. The achievement Yaw $(\psi)$ control occurs by varying the tilt angle $\mu$.

The nonlinear equations of motion of conventional UAVs, which have 6 DOFs, are also used for tri-rotor UAVs. The designed model is free to rotate and translate in 3D space, and the rigid body dynamics are derived by Newton's laws $[10,11]$.

The equations are expressed as follows (see also Table 1).

Force equations:

$$
\begin{gathered}
F_{x}=m(\dot{u}-r v+q w)+m g \sin \theta, \\
F_{y}=m(\dot{v}+r u+p w)-m g \sin \phi \cos \theta, \\
F_{z}=m(\dot{w}+p v-q r)-m g \cos \phi \cos \theta .
\end{gathered}
$$

Moment equations:

$$
\begin{gathered}
L=I_{x x} \dot{p}-q r\left(I_{y y}-I_{z z}\right)-I_{x z} r-I_{x z} p q, \\
M=I_{y y} \dot{q}-p r\left(I_{z z}-I_{x x}\right)-I_{x z}\left(r^{2}-p^{2}\right), \\
N=I_{z z} \dot{r}-p q\left(I_{x x}-I_{y y}\right)-I_{x z} q r .
\end{gathered}
$$

TABLE 1: Formulation notations.

\begin{tabular}{lc}
\hline$F_{X}, F_{Y}, F_{Z}$ & The external forces \\
$L, M, N$ & The external moments \\
$(u, v, w)$ & The translational velocities \\
$(p, q, r)$ & The rotational velocities (angular velocities) \\
$(\phi, \theta, \psi)$ & The rotational angles (Roll, Pitch, Yaw) \\
$\left(I_{X X}, I_{Y Y}, I_{Z Z}\right)$ & The rotational inertias \\
\hline
\end{tabular}

Kinematic equations:

$$
\begin{gathered}
\dot{\phi}=p+q \sin \phi \tan \theta+r \cos \phi \tan \theta, \\
\dot{\theta}=q \cos \phi-r \sin \phi, \\
\dot{\psi}=\frac{(q \sin \phi+r \cos \phi)}{\cos \theta} .
\end{gathered}
$$

Rotation equations:

$$
\begin{aligned}
& \ddot{\phi}=\dot{p}=q r\left(\frac{I_{Y Y}-I_{Z Z}}{I_{X X}}\right)+\frac{L}{I_{X X}} u_{2}, \\
& \ddot{\theta}=\dot{q}=p r\left(\frac{I_{Z Z}-I_{X X}}{I_{Y Y}}\right)+\frac{L}{I_{Y Y}} u_{3}, \\
& \ddot{\psi}=\dot{r}=p q\left(\frac{I_{X X}-I_{Y Y}}{I_{Z Z}}\right)+\frac{L}{I_{Z Z}} u_{4} .
\end{aligned}
$$

2.2. Hovering Flying Mode. Because of the significant balance of tri-rotor UAVs robot in the very beginning, the equilibrium point of hovering state must be figured out. The linearization model is chosen for this duty. Newton-Raphson method, which has the advantage such as converges very fast to root (quadratic convergence), if it converges, it requires only one reliable guess, is the good choice to compute the trim point of system.

Its calculus procedure is that if $x_{i}$ was our last guess and neither $f\left(x_{i}\right)$ nor $f^{\prime}\left(x_{i}\right)$ is zero, then the next approximation derivation will be

$$
x_{i+1}=x_{i}-\frac{f\left(x_{i}\right)}{f^{\prime}\left(x_{i}\right)} .
$$

The four motion equations below are applied by NewtonRaphson method to find out the trim point of tri-rotor UAVs system:

$$
\begin{gathered}
U_{X}=l_{1} b_{1}\left(\Omega_{1}^{2}-\Omega_{2}^{2}\right), \\
U_{T}=b_{1}\left(\Omega_{1}^{2}+\Omega_{2}^{2}\right)+b_{2} \Omega_{3}^{2} \cos \mu, \\
U_{Y}=l_{2} b_{1}\left(\Omega_{1}^{2}+\Omega_{2}^{2}\right)-l_{3} b_{2} \Omega_{3}^{2} \cos \mu, \\
U_{Z}=-l_{4} d_{1} \Omega_{1}^{2}+l_{4} d_{2} \Omega_{2}^{2}+l_{3} d_{3} \Omega_{3}^{2}+l_{3} b_{3} \Omega_{3}^{2} \sin \mu,
\end{gathered}
$$

where $\Omega_{1}, \Omega_{2}$, and $\Omega_{3}$ are the rotor speeds of three rotorcrafts, respectively, $\mu$ is tail tilt angle, $b$ is thrust factor, and $d$ is drag factor. 


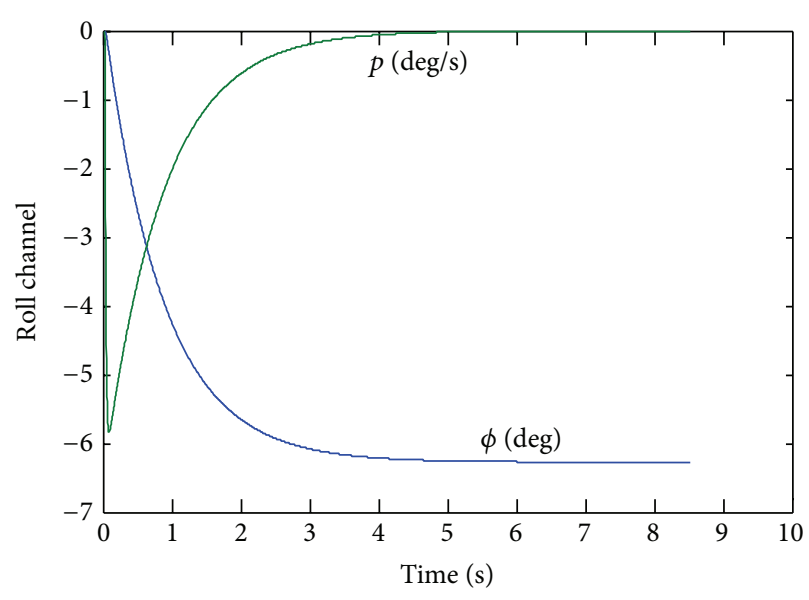

(a) Roll

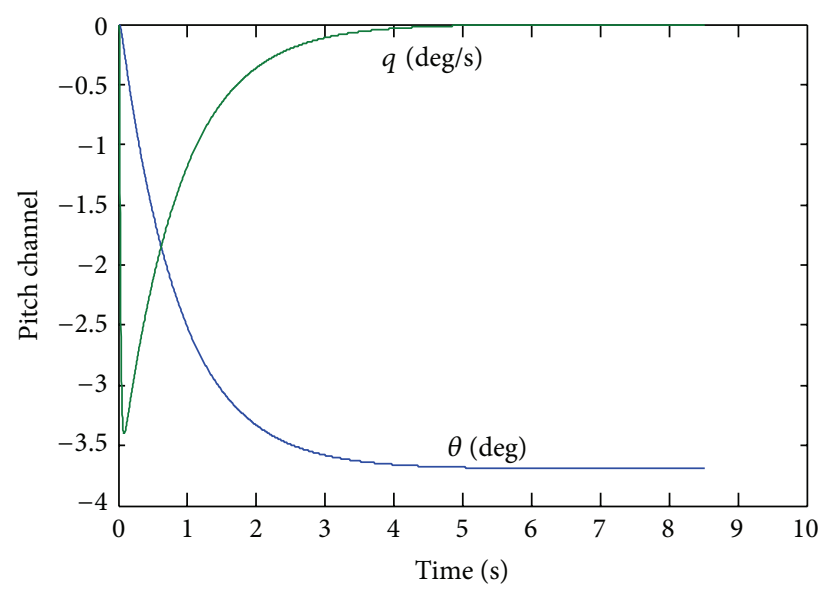

(b) Pitch

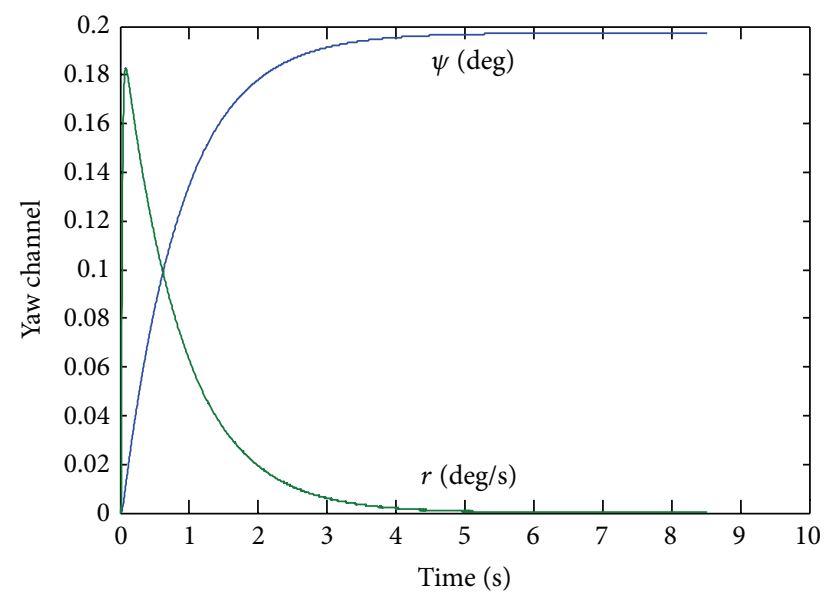

(c) Yaw

FIGURE 2: Linearization model with LQR control gain in each channel.

The thrust $T_{i}=b \Omega_{i}^{2}$ and torque $Q_{i}=d \Omega_{i}^{2}$ are supposed to be constant; that is, these coefficients $b, d$ are not changed. It means that the system is asymptotically stable. Thus, an LQR controller gain is applied to guarantee and maintain the system stability, in both linear and nonlinear models.

\section{Control Strategy Design}

3.1. LQR Control Gain. For the most important thing is to keep hovering mode all the operation time. The system must be asymptotically stable. A system is stabilizable if there exists a state feedback control $u(t)=-k x(t)$ such that the closedloop system is exponentially stable. Next, the Riccati equation is solved for the feedback gain matrix $K: A^{T} P+P A+Q-$ $P B R^{-1} B^{T} P=0$.

If $k=R^{-1} B^{T} P$, then the closed-loop system is asymptotically stable. Thus, the LQR controller gain is applied to guarantee and maintain the system stability, in both linear and nonlinear models (see Figures 2 and 3 ).
3.2. Back-Stepping Control Design. The tri-rotor aerial robot is a lofty nonlinear, multivariable, strongly coupled, and underactuated subsystem. Since the research environment is indoor or low-wind outdoor, in order to achieve flight attitude control of system, back-stepping nonlinear control method is proposed.

The back-stepping controller design usually starts with a "virtual control" input for stabilizing a small subsystem based on Lyapunov stability theorem $[5,8,12,13]$. In order to control various flying attitudes of nonlinear model, the presented controller is applying for the following channels: Roll angle $(-\pi / 2<\phi<\pi / 2)$, Pitch angle $(-\pi / 2<\theta<\pi / 2)$, and Yaw $(-\pi<\psi<\pi)$. In each rotational control, we deal with an easier single-input, single-output (SISO) design problem; each step provides a reference for the next design step. Thus, step by step, a control input for the overall system, which satisfies the Lyapunov candidate function, is designed.

In case of the Roll $[x-\phi]$ channel, for example, the objective of virtual input design is to make the actual state $z_{1}=\phi$ converge to the desired state $z_{2}=\dot{z}_{1}=\phi_{d}$. The 


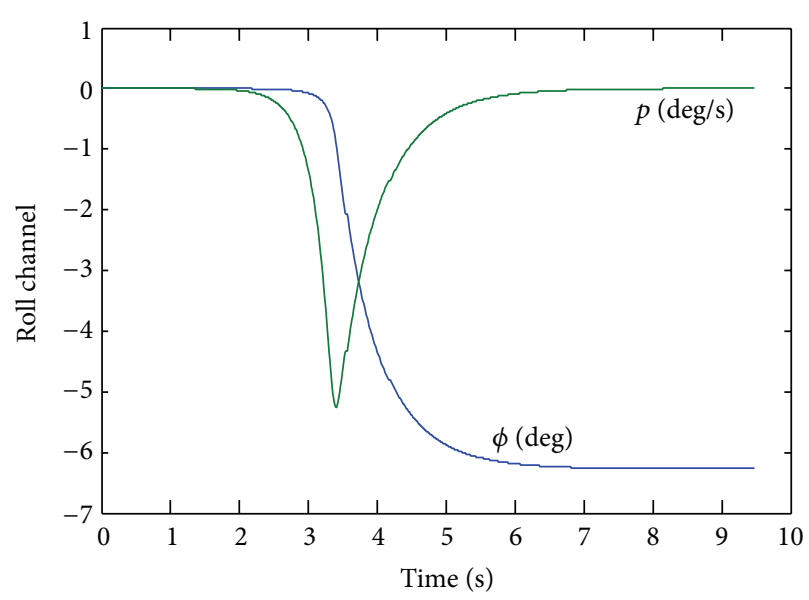

(a) Roll

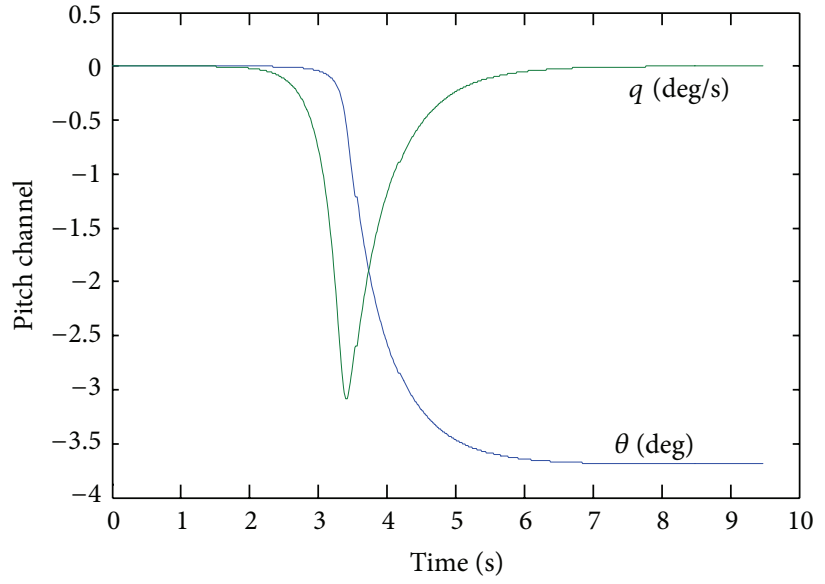

(b) Pitch

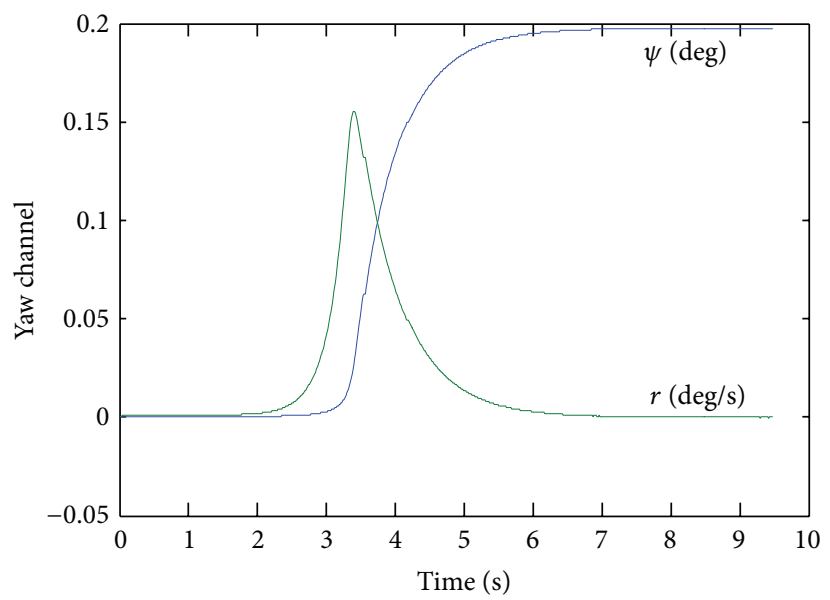

(c) Yaw

FIGURE 3: Nonlinear model with LQR control gain in each channel.

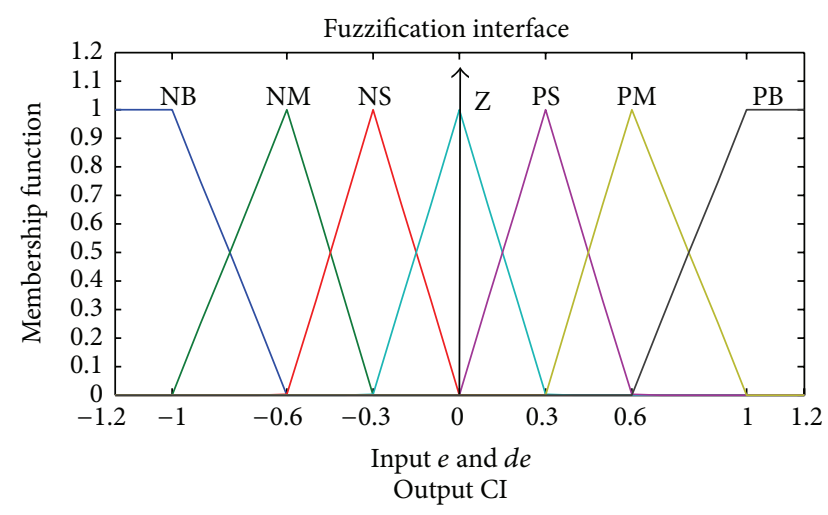

FIGURE 4: Fuzzification interface: input $e$ and $d e$, output CI.

tracking error is defined as $e_{1}=\phi-\phi_{d}$. Assume $v_{1}=\dot{z}_{1}$ is the virtual input for controlling the subsystem state. The chosen Lyapunov candidate function is $V_{1}=(1 / 2) e_{1}^{T} e_{1}>0$.

Let the virtual input $v_{1}=\dot{\phi}_{d}-k_{1} e_{1}$, where $k_{1}>0$ and a regulatable parameter. This makes the subsystem stable since the Lyapunov stability is observed as $\dot{V}_{1}=-e_{1}^{T} k_{1} e_{1}<0$.
Secondly, we will find the stabilizing control law for the whole system while $z_{1}$ converges to $\phi_{d}$. Let the error $e_{2}$ be between $v_{1}$ and $z_{2}$; that is, $e_{2}=z_{2}-v_{1}$. Choose the Lyapunov candidate function for the whole system to be $V_{S}=$ $(1 / 2) e_{1}^{T} e_{1}+(1 / 2) e_{2}^{T} e_{2}>0$.

Then, from (4), the control input will become $u_{2}=$ $\left[-k_{1}\left(\phi-\phi_{d}\right)-k_{2}\left(\dot{\phi}-\dot{\phi}_{d}\right)-q r\left(\left(I_{Y Y}-I_{Z Z}\right) / I_{X X}\right)\left(I_{X X} / L\right)\right]$, where $k_{2}$ is a positive tuning parameter. This makes the Roll channel stable and controllable since the Lyapunov stability is observed as $\dot{V}_{S}=-e_{1}^{T} k_{1} e_{1}-e_{2}^{T} k_{2} e_{2}<0$.

The Pitch channel $[y-\theta]$ with control input $u_{3}$ and Yaw channel $[z-\psi]$ with control input $u_{4}$ are similarly taken as Roll channel. Hence, the whole system is controlled by Lyapunov candidate function presented on back-stepping method.

3.3. Fuzzy Logic Controllers (FLC) Design. The dynamic behavior of FLC is characterized by a set of linguistic rules based on expert knowledge [14-18]. The error $e(t)$ and change-in-error or error rate $d e(t) / d t$ are the input variables; $f$ is the output variable. Fuzzy relations between $e$, $d e$, and $f$ are formed. Then $f$ can be changed on line according to 


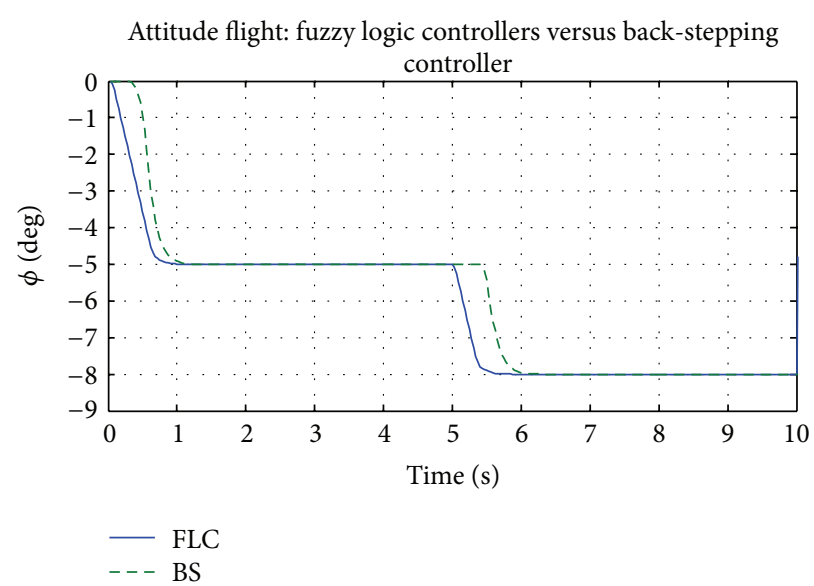

(a) Add disturbance like windy: -3 degree on Roll channel with previous Phi angle $=-5$ degree was controlled stability

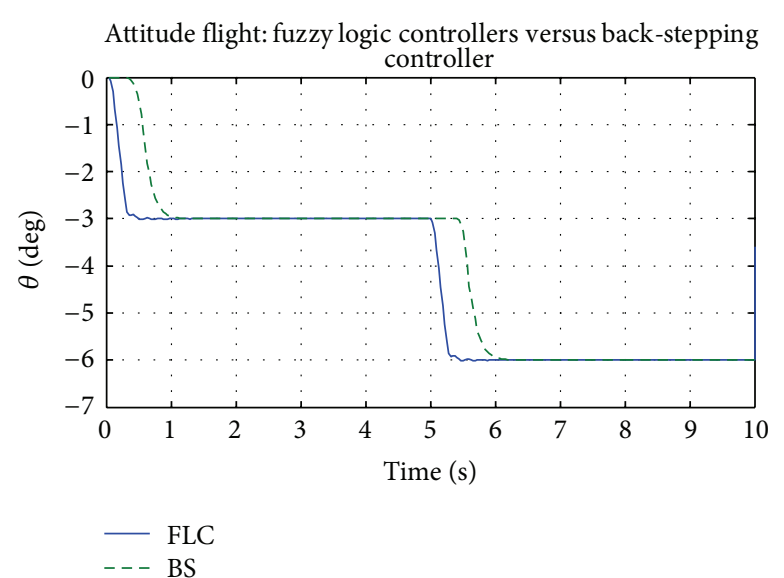

(b) Add disturbance like windy: -3 degree on Pitch channel with previous Theta angle $=-3$ degree was controlled stability

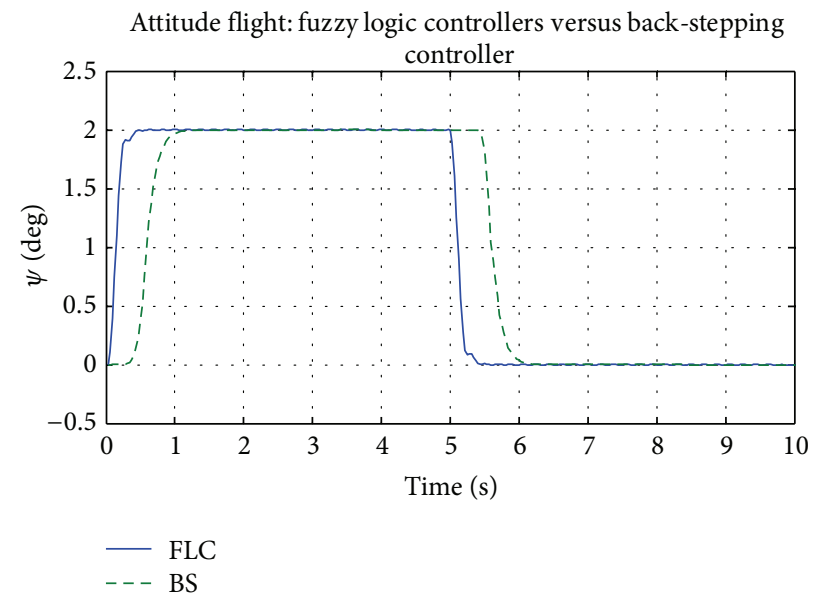

(c) Add disturbance like windy: 2 degree on Yaw channel with previous Psi angle $=-2$ degree was controlled stability

FIGURE 5: Fuzzy logic controllers versus back-stepping controller for each channel: (a) Roll, (b) Pitch, and (c) Yaw, adding disturbance at fifth second.

the rules, current error, and error rate. Figure 4 shows the input/output of fuzzification interface.

Generally, the control output increases in company with the error of angle. Additionally, the controller also judges whether the error increases or decreases and the value of the error difference. It is significant in small error phase that the reversed control output is necessary to ease up the error change when the error decreases sharply.

The linguistics of seven fuzzy triangular membership functions assigned for input and output variables are Negative Big (NB), Negative Medium (NM), Negative Small (NS), Zero (Z), Positive Small (PS), Positive Medium (PM), and Positive Big (PB). The rule for fuzzy controller is shown in Table 2.

\section{Simulations}

The simulation parameters were derived from [6]. However, the $\mu$ tilt angle should be in minus (-) sign of initial condition in order to maintain the system stability. The simulations
TABLE 2: Rule table of fuzzy logic controllers.

\begin{tabular}{cccccccc}
\hline CI & & & \multicolumn{5}{c}{$e(t)$} \\
& NB & NM & NS & Z & PS & PM & PB \\
\hline$d e(t)$ & & & & & & & \\
PB & Z & PS & PM & PB & PB & PB & PB \\
PM & NS & Z & PS & PM & PB & PB & PB \\
PS & NM & NS & Z & PS & PM & PB & PB \\
Z & NB & NM & NS & Z & PS & PM & PB \\
NS & NB & NB & NM & NS & Z & PS & PM \\
NM & NB & NB & NB & NM & NS & Z & PS \\
NB & NB & NB & NB & NB & NM & NS & Z \\
\hline
\end{tabular}

of hovering flight are illustrated in the pilot angle of each channel RPY (Roll, Pitch, and Yaw). It is realized that the hovering state could easily be achieved by equilibrium point. The speeds of each rotorcraft, which are calculated by NewtonRaphson method, are $\Omega_{1}=8418 \mathrm{rpm}, \Omega_{2}=8418 \mathrm{rpm}$, 
TABLE 3: Simulation parameters.

\begin{tabular}{lccc}
\hline Notation & Parameters (unit) & Notation & Parameters (unit) \\
\hline$I_{x x}$ & $0.0057211 \mathrm{~kg} \cdot \mathrm{m}^{2}$ & $l_{1}$ & $0.19 \mathrm{~m}$ \\
$I_{y y}$ & $0.073933 \mathrm{~kg} \cdot \mathrm{m}^{2}$ & $l_{2}$ & $0.122 \mathrm{~m}$ \\
$I_{z z}$ & $0.012545 \mathrm{~kg} \cdot \mathrm{m}^{2}$ & $l_{3}$ & $0.23 \mathrm{~m}$ \\
$b_{1}=b_{2}$ & 0.00013678 & $l_{4}$ & $\sqrt{l_{1}^{2}+l_{2}^{2}}=0.225 \mathrm{~m}$ \\
$d_{1}=d_{2}$ & 0.000024323 & $m$ & $0.84 \mathrm{~kg}$ \\
\hline
\end{tabular}

$\Omega_{3}=8676 \mathrm{rpm}$, and the tilt angle $\mu=-0.0210 \mathrm{rad}(-2.407$ degree). Hovering mode is operated by LQR control gain. Attitude flights controls for each channel are depicted. Furthermore, the results of two more controller strategies are represented. The performance of back-stepping controller for the single tilt tri-rotor has been verified. As found on the simulation results, FLCs give faster response in the very beginning, which means less delay $0.3 \mathrm{sec}$ than back-stepping's. However, the little undershoot occurs when getting the target. The trajectory tracking results demonstrated that the proposed controllers have good pilot performance. All simulations were done by MATLAB/Simulink (see Table 3). Figure 5 shows comparison between fuzzy logic controllers and backstepping controllers for each channel, adding disturbance at fifth second.

\section{Conclusions}

This paper has implemented nonlinear control strategies for the single tilt triple rotor aerial robot. Based on NewtonEuler's laws, the linear and nonlinear mathematical models of tri-rotor UAVs are obtained. Hovering flying mode is achieved by Newton-Raphson numerical analysis method and also controlled by LQR gain. Back-stepping nonlinear controller design is based on constructing Lyapunov candidate function for closed-loop system and has shown the worth results. Furthermore, fuzzy logic controllers have been designed with the human linguistic logic display the even better performance. Effectiveness of the controllers design scheme for UAV tilt tri-rotor is achieved through nonlinear model on each pilot channel.

\section{Acknowledgment}

This work is supported by the National Science Council, Taiwan, China, under Grant nos. NSC 102-2221-E-218-017 and NSC100-2632-E-218-001-MY3.

\section{References}

[1] G. Heredia and A. Ollero, "Detection of sensor faults in small helicopter UAVs using Observer/Kalman filter identification," Mathematical Problems in Engineering, vol. 2011, Article ID 174618, 12 pages, 2011.

[2] Z. Weihua and T. H. Go, "Robust decentralized formation flight control," International Journal of Aerospace Engineering, vol. 2011, Article ID 157590, 13 pages, 2011.
[3] M. W. Spong, S. Hutchinson, and M. Vidyasagar, Robot Modeling and Control, John Wiley \& Sons, Hoboken, NJ, USA, 2006.

[4] D.-W. Yoo, H.-D. Oh, D.-Y. Won, and M.-J. Tahk, "Dynamic modeling and control system design for Tri-rotor UAV," in Proceedings of the 3rd International Symposium on Systems and Control in Aeronautics and Astronautics (ISSCAA '10), pp. 762767, Harbin, China, June 2010.

[5] T. Madani and A. Benallegue, "Backstepping control for a quadrotor helicopter," in Proceedings of the IEEE/RSJ International Conference on Intelligent Robots and Systems (IROS '06), pp. 3255-3260, Beijing, China, October 2006.

[6] S. Yoon, S. J. Lee, B. Lee, C. J. Kim, Y. J. Lee, and S. Sung, "Design and flight test of a small Tri-rotor unmanned vehicle with a LQR based onboard attitude control system," International Journal of Innovative Computing, Information and Control, vol. 9, no. 6, pp. 2347-2360, 2013.

[7] S. S. Cruz and R. Lozano, "Stabilization and nonlinear control for a novel Tri-rotor mini-aircraft," in Proceedings of the IEEE International Conference on Robotics and Automation, pp. 26122617, April 2005.

[8] Y. Yali, J. Changhong, and W. Haiwei, "Backstepping control of each channel for a quadrotor aerial robot," in Proceedings of the International Conference on Computer, Mechatronics, Control and Electronic Engineering (CMCE '10), pp. 403-407, Changchun, China, August 2010.

[9] P. Deuflhard, Newton Methods for Nonlinear Problems, Springer, Berlin, Germany, 1st edition, 2005.

[10] G. D. Padfield, Helicopter Flight Dynamics: the Theory and Application of Flying Qualities and Simulation Modeling, AIAA, 1996.

[11] B. L. Stevens and F. L. Lewis, Aircraft Control and Simulation, Wileys, New York, NY, USA, 1992.

[12] H. K. Khalil, Nonlinear Systems, Publishing House of Electronics Industry, 3rd edition, 2005.

[13] T.-Y. Huang, S.-H. Wu, and H. K. Tran, "Gait control of a biped robot using an exact limit cycle trajectory and the backstepping method," in Proceedings of the Annual Conference (SICE '10), pp. 1819-1824, Taipei, Taiwan, August 2010.

[14] K. M. Passino and S. Yurkovich, Fuzzy Control, Addison-Wesley, Reading, Mass, USA, 1998.

[15] Z. Gao, T. A. Trautzsch, and J. Dawson, "A stable self-tuning fuzzy logic control system for industrial temperature regulation," in Proceedings of the 35th Annual Meeting and World Conference on Industrial Applications of Electrical Energy (IAS '00), pp. 1232-1240, October 2000.

[16] W. Nonnenmacher and Z. Gao, "Fuzzy logic control of an industrial indexing motion application," in Proceedings of the ISA Conference, September 2001.

[17] E. H. Mamdani, "Application of fuzzy algorithms for control of simple dynamic plant," Proceedings of the Institution of Electrical Engineers, vol. 121, no. 12, pp. 1585-1588, 1974.

[18] E. H. Mamdani, "Application of fuzzy logic to approximate reasoning using linguistic synthesis," IEEE Transactions on Computers, vol. C-26, no. 12, pp. 1182-1191, 1977. 


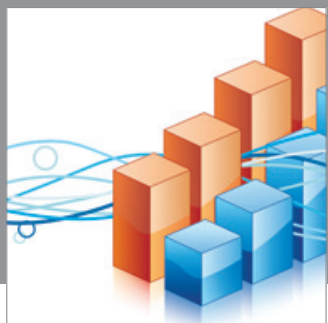

Advances in

Operations Research

mansans

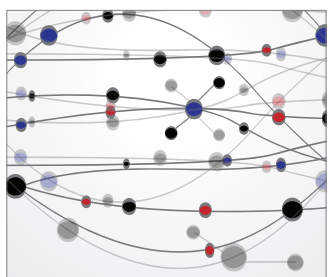

The Scientific World Journal
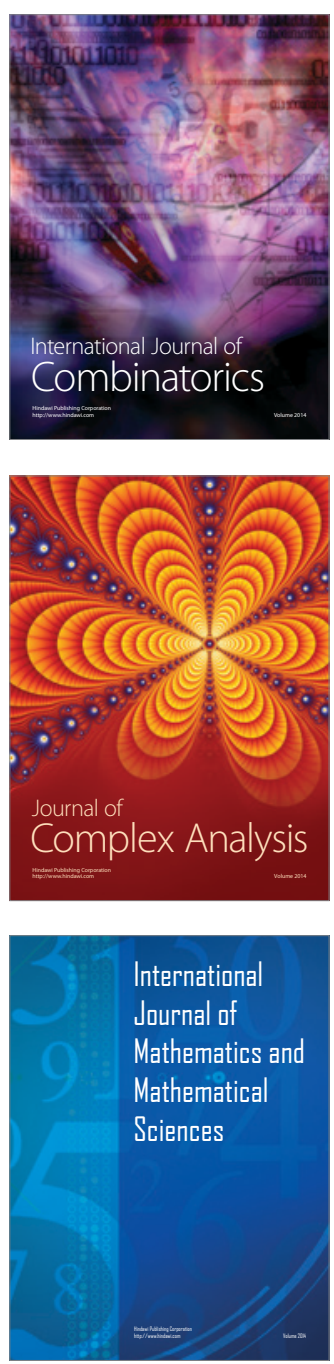
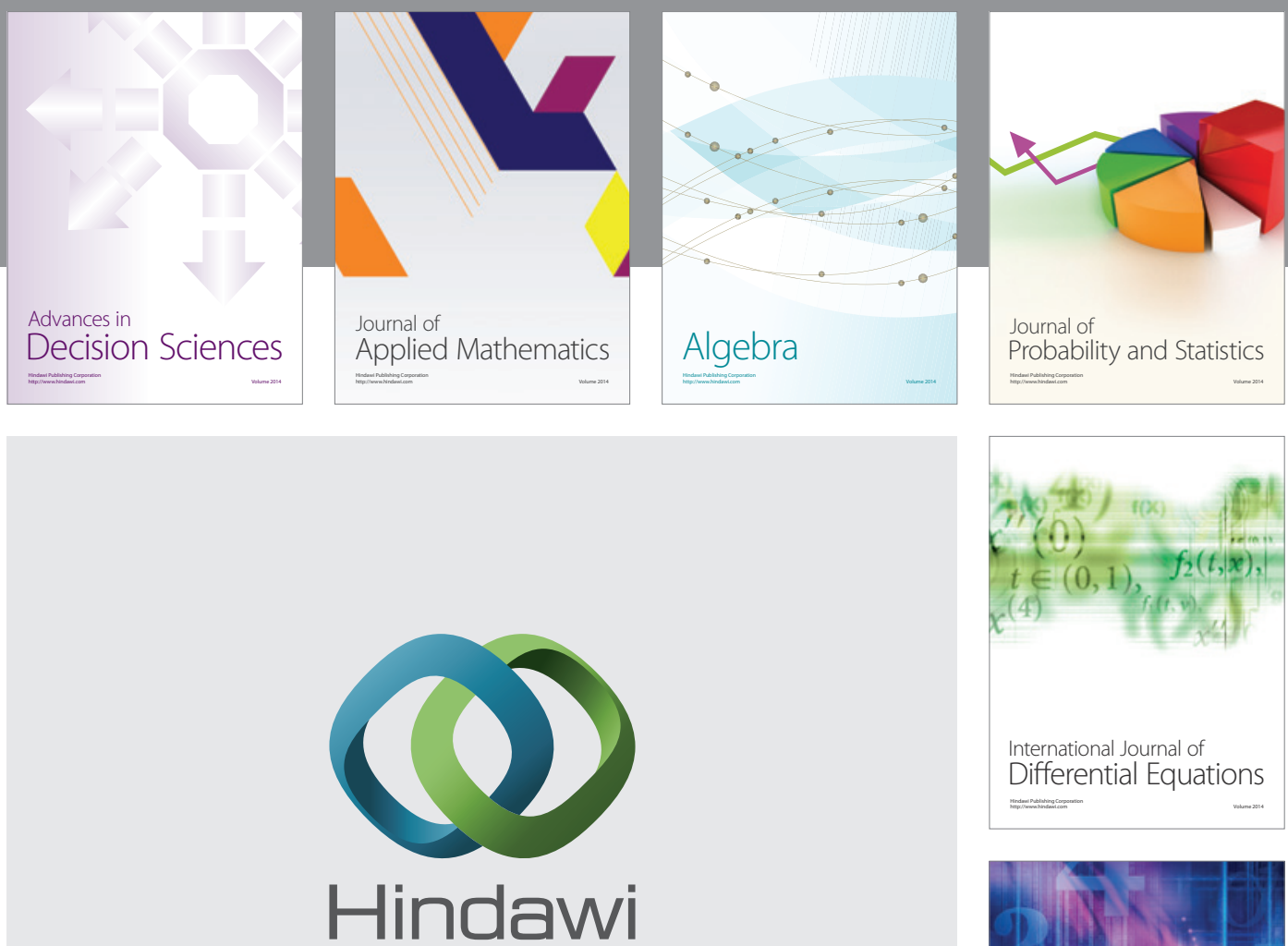

Submit your manuscripts at http://www.hindawi.com
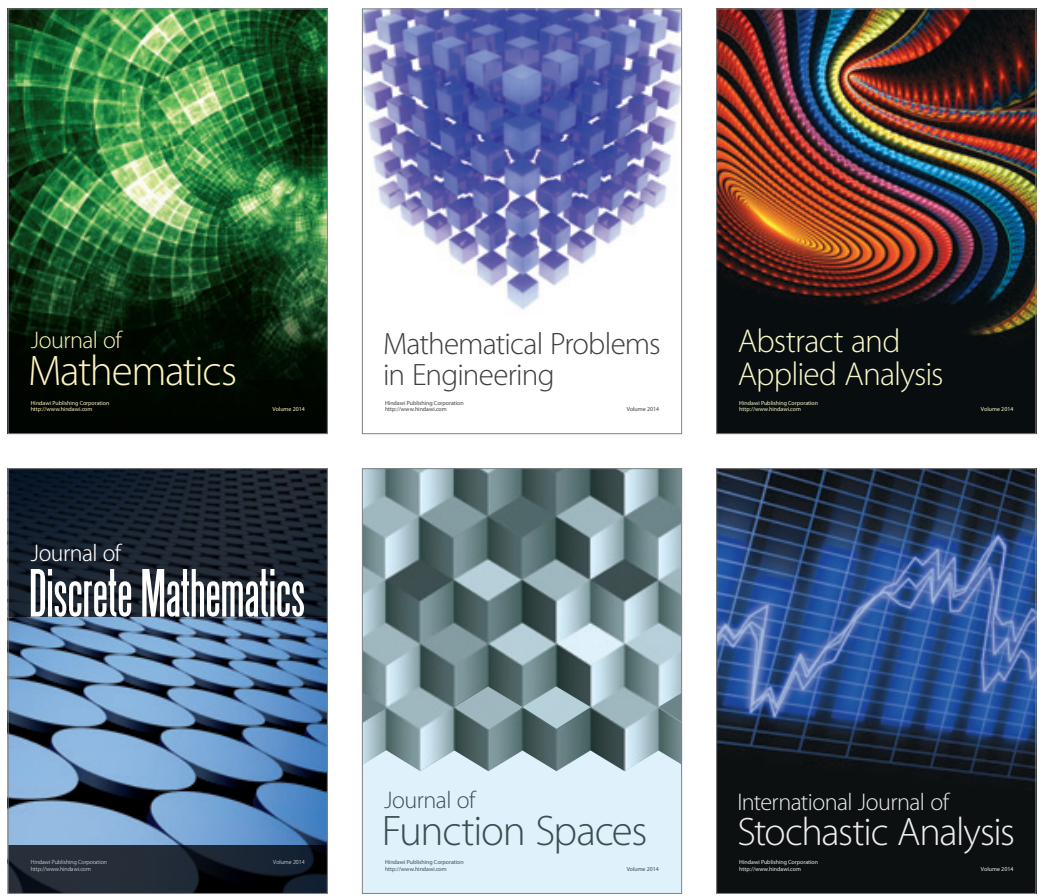

Journal of

Function Spaces

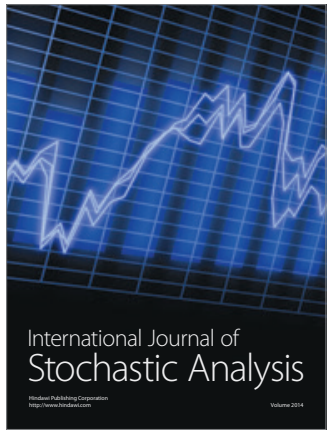

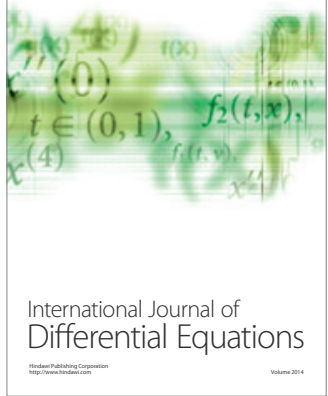
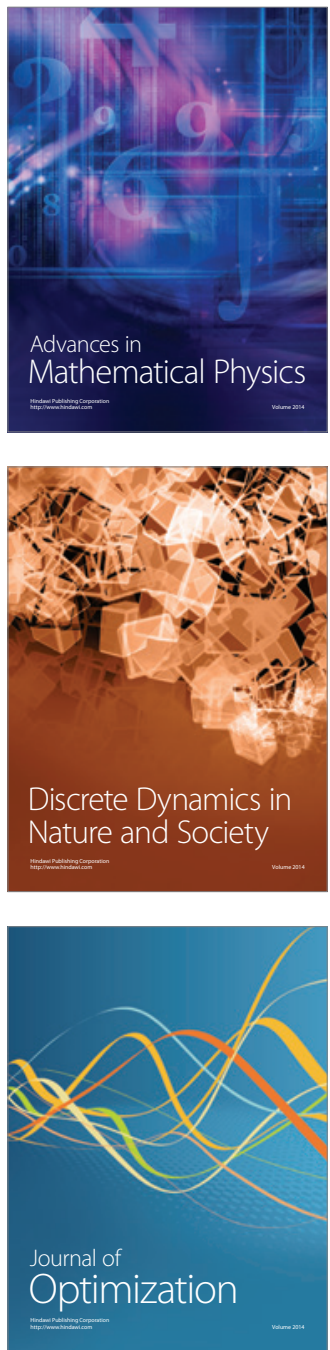